\title{
Modeling of a Mill for Processing a Turbine Blade
}

\author{
Julia Malneva ${ }^{1, *}$, Vadim Kuts $^{1}$, Aleksandr Altukhov ${ }^{1}$, Luibov Kuznetsova ${ }^{1}$, Konstantin \\ Kuznetsov $^{1}$ and Evgenii Vorobiev ${ }^{1}$ \\ ${ }^{1}$ Southwest State University, 94, 50 let Oktyabrya Avenue, 305040 Kursk, Russia
}

\begin{abstract}
The aim of the study is to model the cutting edges, front and rear surfaces of the cutter with a constructive feed for machining a turbine blade. In the modeling process, we used the method of compiling mathematical models of technological processes for obtaining surfaces of parts using cutting. As a result, equations of the cutting edge of the mill, equations of the producing surface were obtained, parametric equations of the rear and front surfaces of the cutting teeth were constructed, and it was also proposed to introduce corrections to reduce the profile distortion after regrinding into the insertion motion parameter.
\end{abstract}

\section{Introduction}

An important step in the design process of a shaped cutter with a variable radius is the modeling of the cutting elements of the tool.

The form of the cutting edge equation depends on the type of the front surface (flat, helical) and the angle of inclination of the flat front surface $(\omega)$.

The final solution is not the cutting edge equation, but an array of coordinates, because the solution was presented not in an analytical form, but in numerical form.

For the studied case of a curved front surface, the cutting edge is the result of the intersection of the producing surface with a plane passing through the axis of the tool rotation and located at a certain angle $\theta$ with respect to the $\mathrm{X}$ axis. The resulting edge does not have an inclination angle $\omega$ and belongs to the same plane of the radial section of the cutter, while it is not linear.

\footnotetext{
*Corresponding author: yuliyamaximencko2010@yandex.ru
} 


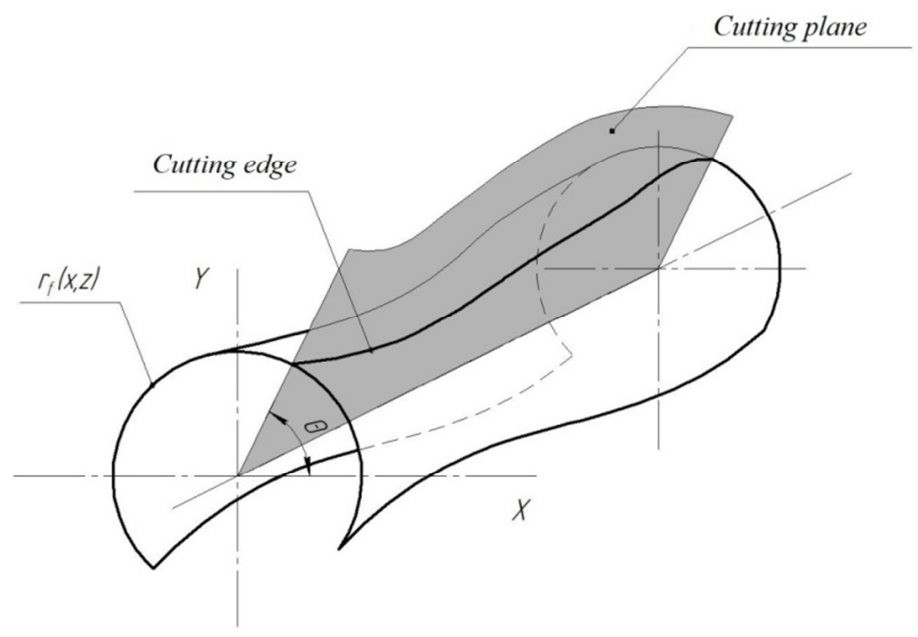

Fig. 1. Cutting edge as a result of the intersection of the secant plane with the tool surface.

\section{Materials and Methods}

The equation of the leading surface of the cutting edge can be obtained by solving the equation with respect to the parameters $\theta$ and $\mathrm{z}$ of the vector $\overline{\mathrm{r}_{\mathrm{f}}}$. In order to express this equation, it is necessary to perform the following steps:

We express the equation of the front surface using the installation vector:

$$
\bar{r}_{\Pi i}=A_{\Pi i} \cdot A_{u s t} \cdot e^{4},
$$

where $\overline{r_{\Pi l}}$ - the equation of the front surface plane of the i-th cutting edge in its own coordinate system;

$e^{4}=\left[\begin{array}{llll}0 & 0 & 0 & 1\end{array}\right]^{T}-$ unit vector of zero length;

$A_{\Pi i}$ - installation matrix of the front surface plane:

$$
A_{\Pi i}=A^{6}(\gamma) \cdot A^{2}(b) \cdot e^{4}
$$

where $A^{2}(b)$ - motion matrix relative to the coordinate $y$;

$A^{6}(\gamma)$ - rotation matrix taking into account the rake angle of the cutting edge;

$\theta_{i}$ - a parameter defining the angular position of the cutting edge;

$\gamma-$ rake angle.

$A_{\text {ust }}$ - installation matrix of the front surface plane, which can be defined as (Fig. 2)

$$
A_{u s t}(\theta, z)=A\left(\bar{k}_{k_{f}}, \bar{j}_{k_{f}}, \bar{r}_{k_{f}}\left(\theta_{j_{k}}, z\right)\right),
$$

where $A-$ a function that determines the position of the axes represented in the coordinate system of the cutter;

$\bar{k}_{k_{f}}-$ vector, defining the position of the axis $Z_{x}$, represented in the coordinate system of the cutter

$$
\bar{k}_{k_{f}}=\left[\begin{array}{llll}
0 & 0 & 1 & 0
\end{array}\right]^{\mathrm{T}},
$$

$\bar{j}_{k_{f}}$ - vector, defining the position of the axis $Y_{3}$, represented in the coordinate system of the cutter

$$
\bar{j}_{k_{f}}=\left.\bar{r}_{f_{j k}}\left(\theta_{j_{k}}, z\right)\right|_{X_{f j} Y_{f j}}
$$


$\bar{r}_{f_{j k}}\left(\theta_{j_{k}}, z\right)$ - vector defining the position of the origin of the coordinate system of the rear surface in the coordinate system of the cutter

$$
\bar{r}_{f_{j k}}\left(\theta_{j_{k}}, z\right)=\left.\overline{r_{f_{j}}}(x, z)\right|_{x=f\left(\theta_{k_{j}}\right)} .
$$

where $\theta_{i}$ - a parameter defining the angular position of the cutting edge;

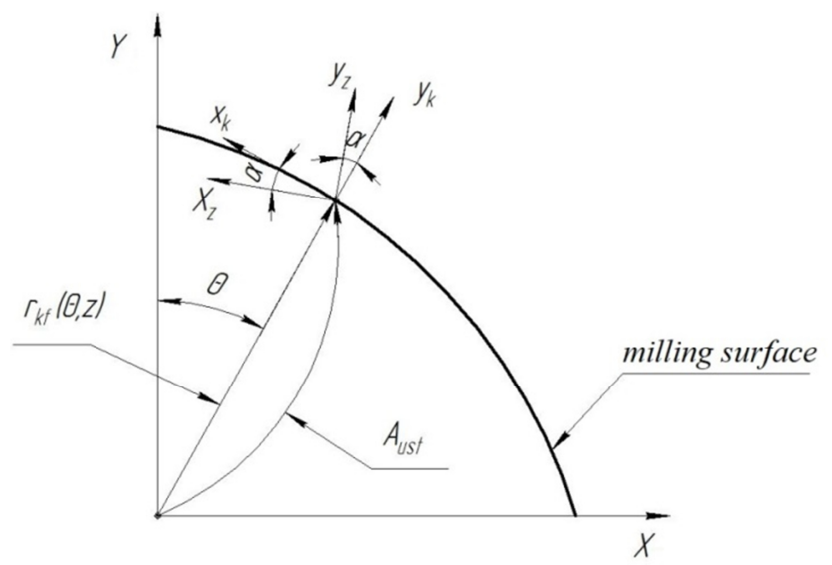

Fig. 2. Rear surface plane installation diagram.

Also for the back surface:

$$
\bar{r}_{3 i}=A_{\text {зді }} \cdot A_{\text {up }} \cdot e^{4}
$$

where $\overline{r_{3 l}}$ - the equation of the front surface plane of the i-th cutting edge in its own coordinate system;

$A_{\text {зді }}$ - back plane adjustment matrix

$$
A_{0}=A_{3 i} A^{6}(\alpha) \cdot A^{2}(b)
$$

$A^{6}(\alpha)$ - rotation matrix taking into account the back corner of the cutting edge;

$A_{3 \mathrm{i}}-$ installation matrix of the plane of the rear surface at $\alpha=0$;

$\alpha-$ back corner.

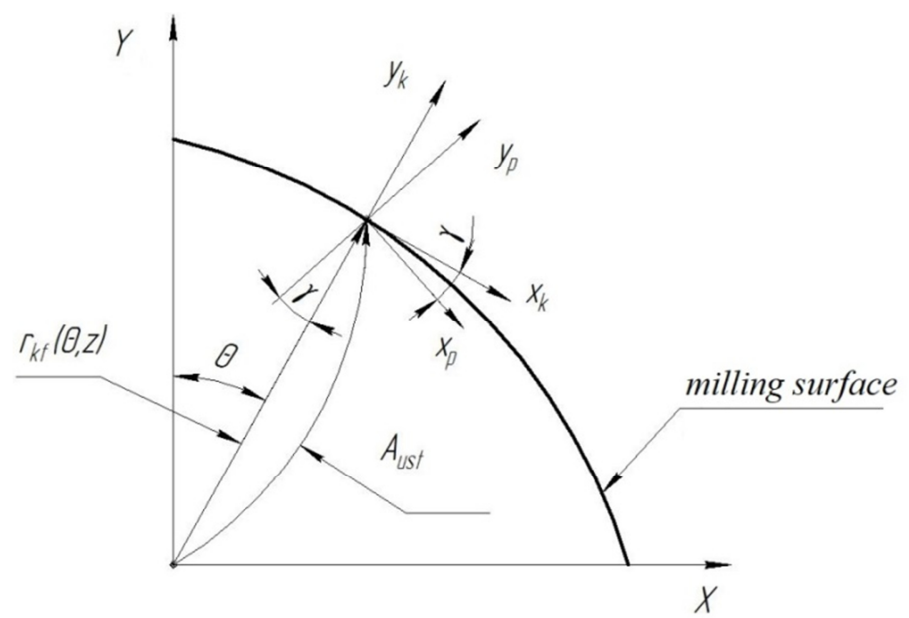

Fig. 3. Front surface plane installation diagram. 


\section{Results}

Based on the obtained solutions, it becomes possible to simulate the normal to the front and back surfaces.

Due to the fact that a straight cutting edge with $\omega=0$ is simpler and cheaper to manufacture in the future, we will build the model for this particular option.

The coordinates of the resulting tool surface depend on the coordinates of the sections of the part. The purpose of the calculation is to create cutting edges whose angular pitch will not depend on these coordinates.

We create an array of angles, that is, we break the surface into edges, where each edge has its own angular position. Then for each edge we create, expressing its equation [1-5].

To begin with, solving the equation of arccosines, we display the values of the angles of the planes passing through the origin and a point on the curve of the first section of the tool (Fig. 4).

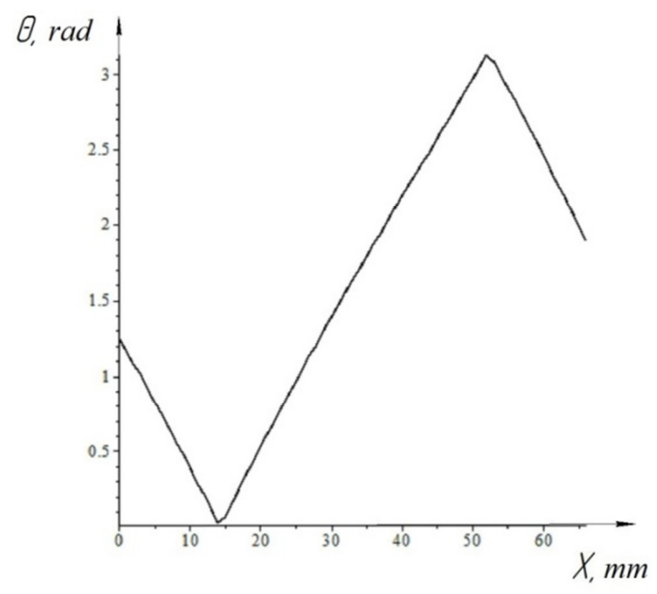

Fig. 4. Angles after solving the equation.

As can be seen from the obtained graph, a correction is necessary in solving this expression to ensure a monotonic change in the values of the angles. Therefore, we start the process of converting angles, which will choose a solution that does not violate the construction logic (Fig. 5). 


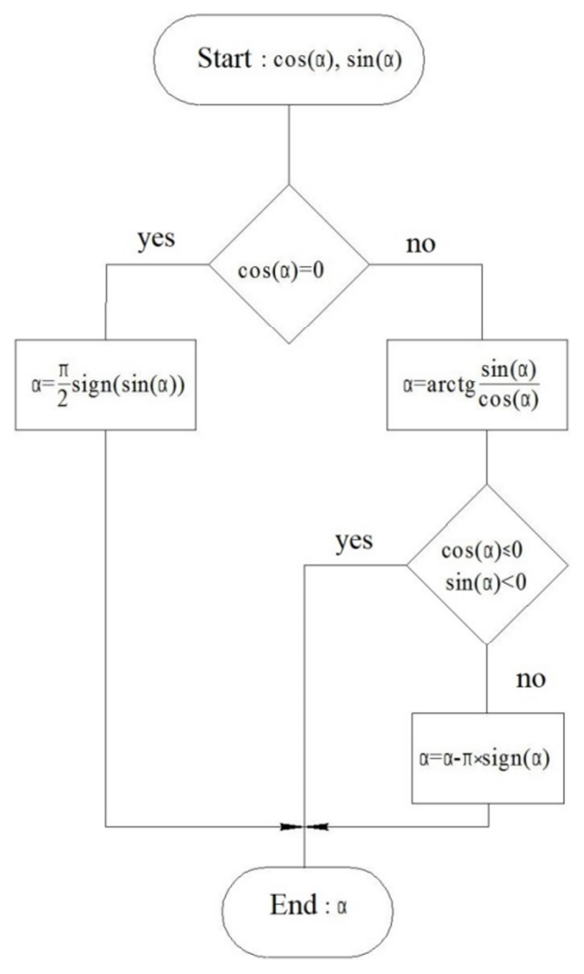

Fig. 5 The correction cycle of the equation solutions.

We display the values of the angles of the planes passing through the origin and the point on the curve of the first section of the tool, taking into account the adjustment (Fig. $6)$.

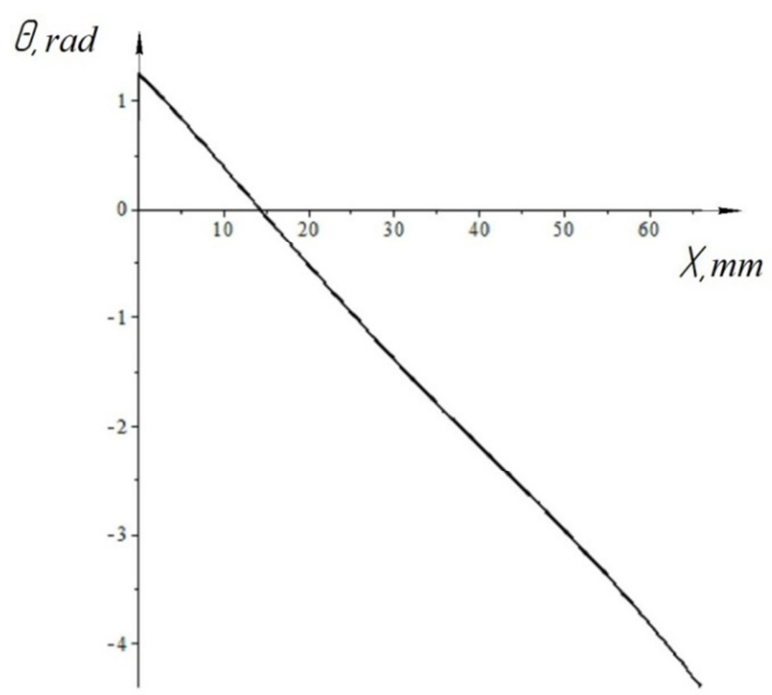

Fig. 6. Angle values after adjusting the solutions of the equation. 


\section{Discussion}

To set the edge in the secant plane, it is necessary to deprive the angles depending on the coordinates of the blade sections. To do this, we move from the angular position of the point on the first section of the tool surface to the new coordinates, which will be in the same plane relative to the coordinate system of the tool surface (Fig. 7).

Then we express the surface function through the obtained coordinates, thereby obtaining the equation of the cutting edge.

$$
R_{r f k} 2(x, z) \rightarrow R_{r f k}\left(x\left(\theta_{k}\right), z\right)
$$

Next, we set the calculation function of the matrix elements, vector modules to determine the position of the axes represented in the coordinate system of the cutter.

Using the obtained function, we create the installation matrix of the front and rear surfaces of the cutting tooth, and set the rear and front angles: $\gamma=20^{\circ}$ and $\alpha=15^{\circ}$; of the surfaces equation relative to the coordinate system, created by the installation matrices.

Enter the values of the front and rear angles:

$$
\mathrm{A}_{u s t}(\theta, z)=A\left(\bar{k}_{k_{f}}, \bar{j}_{k_{f}}, \bar{r}_{k_{f}}\left(\theta_{j_{k}}, z\right)\right) .
$$

$$
\begin{aligned}
& \gamma=20^{\circ} ; \\
& \alpha=15^{\circ} .
\end{aligned}
$$

We express the equations of the front and rear planes:

$$
\begin{gathered}
r_{p}(\theta, v, \gamma)=A_{u p}(\theta, z) A_{6}(-\gamma) A_{2}(v) e_{4} \\
r_{z}(\theta, v, \alpha)=A_{u p}(\theta, z) A_{6}(\alpha) A_{2}(v) e_{4} .
\end{gathered}
$$

Next, we calculate the angles in the first section of the tool through an equal step relative to the coordinates of the given sections of the scapula.

$$
\theta_{i j}=\operatorname{arctg}\left(R_{r f}(x, 1)\right)
$$




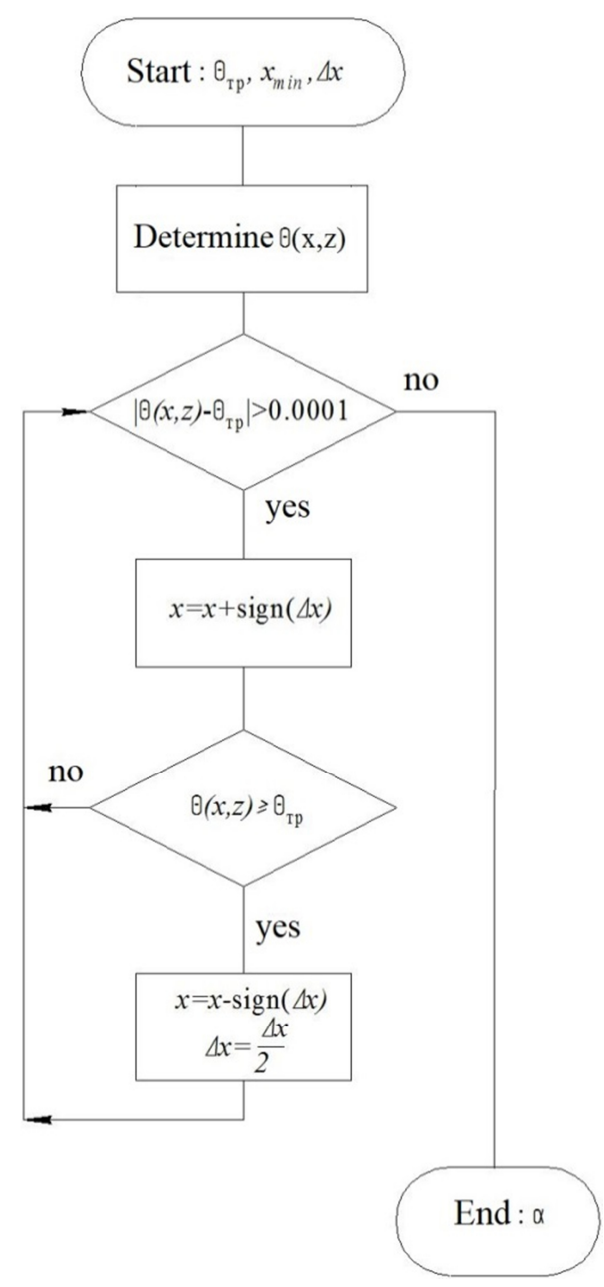

Fig. 7 Coordinate transformation cycle

Regarding the maximum and minimum angles, we calculate the angular step, assuming that the number of teeth is equal $Z_{f}=23$. Then, using this step, we calculate the angles.

$$
\begin{gathered}
\theta_{\min }=1.25 ; \\
\theta_{\max }=-4.39 ; \\
\Delta \theta=\frac{\left|\theta_{\max }-\theta_{\min }\right|}{Z_{f}}, \\
\theta=0.25
\end{gathered}
$$

Adding the step size to the minimum angle, we obtain the angles of arrangement of the cutting teeth [6-9]. 


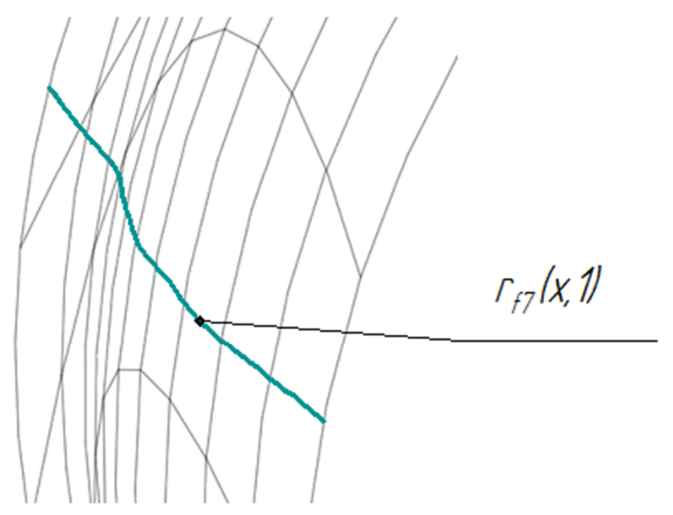

Fig. 8. The cutting edge on the tool surface.

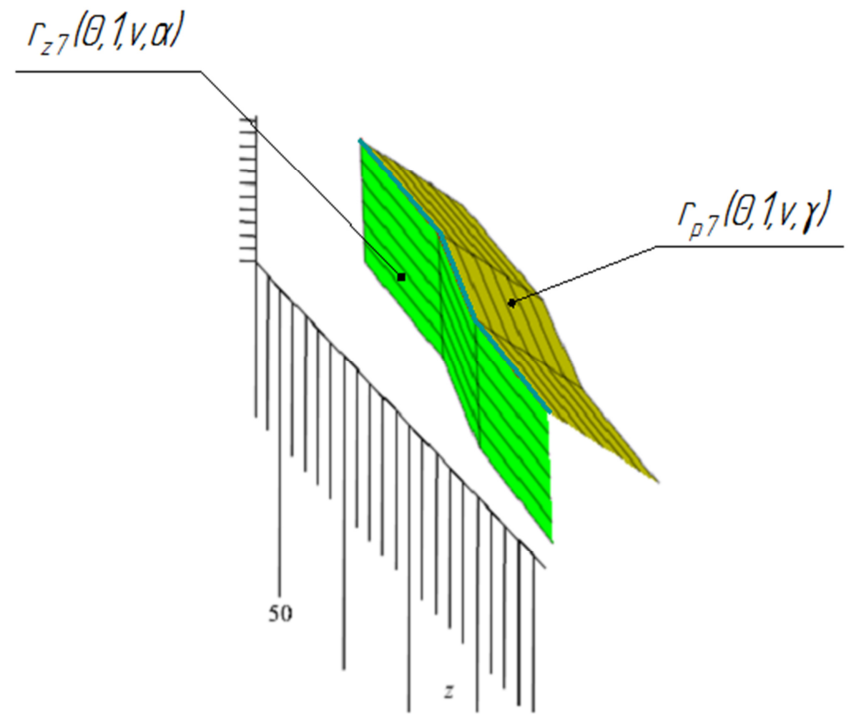

Fig. 9. Front and back surfaces of cutting tooth cutters.

\section{Conclusions}

From the obtained images it is seen that the rear and front surfaces are not flat, that is, the rear and front angles are variable. The cutting edge itself lies in the plane, but is not straight [10-15].

This work was supported by a scholarship from the President of the Russian Federation for 2019-2021. Project number - SP-2950.2019.1.

\section{References}

1. Y. Maksimenko, V. Kuts, J.R. Russian Engineering Research. 34, 785-788 (2014)

2. V. Kuts, Y. Malneva, O. Fedonin, Procedia Engineering. 1120-1126 (2017)

3. V. Kuts, Y. Malneva, V. Skantsev, Procedia Engineering. 696-701 (2016) 
4. E. Ageev, A. Altukhov, L. Kuznetsova, Y. Malneva, Revista Publicando. 5, 17, 459465 (2018)

5. Y. Malneva, A. Altukhov, E. Vorobiev, L. Kuznetsova, International Journal of Advanced Science and Technology. 28, 1, 95-103 (2019)

6. E. Ageev, A. Altukhov., A. Novikov, MATEC Web of Conferences. 01124 (2018)

7. E. Ageev, A. Altukhov., A. Novikov, MATEC Web of Conferences. 01125 (2018)

8. E. Ageev, A. Altukhov., E. Ageeva, S. Khardikov, IOP conference series: materials science and engineering. 032002 (2018)

9. E. Ageev, A. Altukhov, V. Kozlikin, R. Latypov, IOP Conference Series: Materials Science and Engineering. 032004 (2018)

10. R. Latypov, E. Ageev, A. Altukhov, E. Ageeva, Russian metallurgy (Metally). 6, 573575 (2018)

11. L. Loeber, S. Biamino, U. Ackelid, Solid freeform fabrication proceedings. $11547-$ 556 (2011)

12. D. Gu, W. Meiners, K. Wissenbach, R. Poprawe, International Materials Reviews. 57 133-164 (2012)

13. E. Ageev, A. Shcherbakov, A. Novikov, Journal of fundamental and applied sciences. 1152-1157 (2016)

14. E. Ageev, A. Altukhov., S. Hardikov, Journal of nano- and electronic physics. 4, 04055-1 - 04055-3 (2016)

15. J. Karlsson, A. Snis, H. Engqvist, J. Lausmaa, Journal of Materials Processing Technology 213 2109-2118 (2013) 